\title{
MINIMAL TEMPLATE SIZE FOR IRIS-RECOGNITION
}

\author{
R. Sanchez-Reillo, C. Sanchez-Avila, J.A. Martin-Pereda \\ e-mail: reillo@tfo.upm.es \\ E.T.S. Ingenieros de Telecomunicacion \\ Universidad Politécnica de Madrid \\ Ciudad Universitaria, $\mathrm{s} / \mathrm{n}$ \\ 28040 MADRID. SPAIN
}

\begin{abstract}
A method to achieve improvement in template size for an iris-recognition system is reported. To achieve this result, the biological characteristics of the human iris have been studied. Processing has been performed by image processing techniques, isolating the iris and enhancing the area of study, after which multi resolution analysis is made. Reduction of the pattern obtained has been obtained via statistical study.
\end{abstract}

Key words: iris recognition, multi resolution analysis.

\section{INTRODUCTION}

The growing usage of databases and telecommunication networks for storing and transmitting health records, makes necessary the application of automatic methods of user authentication to access such sensible data. The means already used for this purpose nowadays go from Personal Identification Numbers (PIN's) or passwords to smart cards. But none of these methods do really identify a person, but only the knowledge of some data or belonging of a determined object. The only way to identify a person with sufficient legal background is biometric identification, and from all the techniques that exist nowadays (voice, fingerprint, etc.) iris recognition is the most promising for high security environments.

Several studies have shown that normal variations in colouring and architecture of the tissues of the iris are so multitudinous that there are not ever two irises alike, not even for uniovular (identical) twins [1]. Even for a single person his two irises are also different. This leads to the fact that in an iris recognition system, the False Acceptance Rate (the probability of an intruder entering the system) is null. And through good processing algorithms and optimal selection of the template for each user, a False Rejection Rate (the probability of an authorised user to be rejected by the system) is below $10 \%$ for a single try.

A method to improve the template size for an iris-recognition system, as a function of the False Acceptance Rate desired, will be the objective of this paper.

\section{METHOD AND RESULTS}

As the first process, the iris location and isolation is performed. This is perfonned taking profit from the circular pattem of the iris within the eye studying the first derivate of the intensity of the image around a circle with fixed centre and variable radiuses:

$$
\frac{\partial}{\partial r} \oint_{x_{i n}, y_{0}, r} \frac{I(x, y)}{2 \pi r} d s
$$

The same process is performed to eliminate the pupil from the iris. Once isolated, the resuiting image is stretched for better processing. Then wavelet multi resolution analysis is carried out based on Gabor filtering [2] trough polar coordinate system:

$$
G(r, \theta)=e^{-i \omega\left(\theta-\theta_{0}\right)-\frac{\left(r-r_{0}\right)^{2}}{\alpha^{2}}-\frac{\left(\theta-\theta_{0}\right)^{2}}{\beta^{2}}}
$$

After obtaining the wavelet coefficients, a reduced set of them has been obtained through main component analysis. With the set of data chosen, a primary pattern is formed. This pattern is used in three statistical decision schemes for the verification process: Euclidean and Hamming distances and Gaussian Mixture Modelling (GMM) [3]. In this application, distances between authentic eyes and imposters are so different (around 0.1 for authentics and 0.5 for imposters), that a universal threshold could be applied, being sufficient the usage of Euclidean and Hamming distances instead of GMMs, with the advantage of lower computational cost of the two formers compared with the latter.

Once developed the recognition algorithm, a new reduction has been performed obtaining different False Rejection Rates as a function of that pattern size. This leads to a pattern (or a template ) size different for each degree of security and user comfort desired. Final figures for this function have been obtained by increasing the iris database size.

\section{REFERENCES}

[1] M.L. Berliner, "Biomicroscopy of the Eye". Paul B. Hoeber, Inc. 1949

[2] J. G. Daugman, "High Confidence Visual Recognition of Persons by a Test of Statistical Independence". IEEE Trans. PAMI, vol. 15, no. 11, Nov. 1993.

[3] R. O. Duda, P. E. Hart, "Pattern Classification and Scene Analysis". John Wiley \& Sons. 1973. 